\title{
ADVANCED ELECTRODE DESIGN FOR ELECTROSTATIC PRECIPITATORS
}

By: S. Bernstein, K. Ushimaru, E. W. Geller

Flow Research Company

2141468 th Avenue South

Kent, Washington 98031

\section{MASTER}

\section{ABSTRACT}

The subject of this paper is a wavy electrode precipitator which employs a new collecting electrode. The wavy electrode precipitator incorporates a contoured collecting electrode geometry which produces a nearly uniform electric field along the electrode. This feature allows operation with higher average electric field strengths near the electrode than in conventional designs. The wavy electrode provides separated flow zones within its "valleys", which fluid dynamically shield particles from the main stream. The design also provides mechanical rigidity to vertical bending wtihout the sharp flanges normal to the flow commonly found in conventional precipitators. These features permit increased performance for all particle sizes, but especially help in the precipitation of fine particles which are normally the most difficult to collect.

\section{INTRODUCTION}

This paper describes the wavy electrtode precipitator and gives an analysis of its performance. This advanced electrode design is based on a unique geometrical configuration of electrodes which is expected to provide significantly improved performance as well as reduced cost over conventional precipitators.

Overall reviews of electrostatic precipitator technology are given by White $(1,2)$, and Robinson (3). A state-of-the-art precipitator configuration is illustrated in Figure la. The collecting electrodes are typified by baffles which provide plate stiffness and shield the collected particulate layer from direct scouring by the mainstream, particularly during rapping. The advanced design presented in Figure $1 \mathrm{~b}$ is typified by a wavy collecting electrode shape which provides the same functions with certain advantages. A brief outline of fundamental collection processes is given here to emphasize the advantages of the latter configuration. These processes are ion generation, particle charging, particle capture, particle removal, particle reentrainment, and sparkover.

Gas ions are obtained from a corona discharge at the anode to provide a source for particle charging by diffusion and by field charging. Diffusion charging dominates the charging process for very small particles (submicron 


\section{DISCLAIMER}

This report was prepared as an account of work sponsored by an agency of the United States Government. Neither the United States Government nor any agency Thereof, nor any of their employees, makes any warranty, express or implied, or assumes any legal liability or responsibility for the accuracy, completeness, or usefulness of any information, apparatus, product, or process disclosed, or represents that its use would not infringe privately owned rights. Reference herein to any specific commercial product, process, or service by trade name, trademark, manufacturer, or otherwise does not necessarily constitute or imply its endorsement, recommendation, or favoring by the United States Government or any agency thereof. The views and opinions of authors expressed herein do not necessarily state or reflect those of the United States Government or any agency thereof. 


\section{DISCLAIMER}

Portions of this document may be illegible in electronic image products. Images are produced from the best available original document. 
size); the charge increases as the free ion density increases. High ion density in turn is obtained by high operating voltage and uniform current distribution in the interelectrode space. Field charging occurs when a collision of an ion and a particle takes place. A particle continues to capture ions until the repulsive electric field of the accumulated free ions on the particle balances the attracting electric field of the background. The value of the saturation charge increases linearly with the magnitude of the background electric field, which is determined by the voltage applied to the electrodes and by the electrode configuration.

An electrically charged particle experiences a coulomb force directed toward the collecting electrode and proportional to the charge on the particle and the intensity of the electric field. Since this force increases with particle charge and with the driving electric field intensity, highly charged particles and a high average field intensity along the collecting electrode surface promote efficient collection. The resulting migration accelerations for fine particles are small compared to the accelerations imparted by the turbulent fluctuations present in the flow through the precipitator. However, in regions near the collecting electrode where the flow is separated and relatively protected from the turbulent eddies, the electric field forces are effective in moving the particles to the collector. Thus, one design goal is to increase the sparkover threshold (discussed below), which is the determining factor for the maximum voltage that can be applied. Another goal is to enhance the charging and collecting electric field and current distribution for a given operating voltage which will also increase the charging effectiveness.

It is desirable to maximize both the charging and the collecting field operation at the highest possible voltage. The upper limit for the operating voltage is determined by the sparkover voltage of the given electrode. Thus, peaks in the distribution of electric field intensity along the collecting electrode surface tend to promote early sparkover. Conversely, a uniform distribution delays the onset of sparkover.

Several other phenomena play significant roles in precipitator performance (e.g., chemical composition of the particulate, back corona generation, the conveyance of particulates into the hopper, and particulate removal). We do not intend to minimize the significance of these process, although a full discussion of these topics is beyond the scope of the paper.

\section{THE WAVY ELECTRODE PRECIPITATOR}

The wavy electrode precipitator is illustrated in Figure $1 \mathrm{~b}$. In terms of electric field and gas flow characteristics, the fundamental improvements over the standard precipitator configuration shown in Figure la are: (1) A more uniform electric field which gives a higher average electric field for a speclfied operating voltage and which allows operation at a higher operating voltage without sparkover. (2) The ability to obtain this condition while maintaining separated flow regions which, for the standard configuration, are created by flanges that are generally detrimental to the electric field. The bulging of the flow channel at each of the wire electrodes provides the more uniform electric field (1.e., voltage gradient) and also provides the pockets 
of "dead" air. (3)The wave shape provides structural stiffness for the collector plates so that no stiffeners need be attached during manufacture.

The disadvantages and unknowns associated with the wavy electrode precipitator are the increased cost of manufacturing the wavy shape, the problem of how to design the hanging of plate so as not to promote sparkover, and the fraction of the collector plate area that will be protected from scouring by the malnstream as compared to a standard configuration. The theoretical investigation has provided some quantitative measure of the improvement in collection efficiency that can be expected from the wavy electrode configuration. This work is reported in the next section.

\section{PERFORMANCE ANALYSIS}

In order to compare the performance of the advanced wavy electrode with that of the conventional electrode, a detailed analysis of fluid dynamic flow field, electrical characteristics and efficiency was performed. The analysis included the adaptation of a numerical model introduced by Bernstein et al., $(4,5)$ which consists of a system of seven coupled nonlinear partial differential equations: a three-equation fluid dynamics model which describes conservation of mass and momentum for the fluid flow, a two-equation turbulence model, and a two-equation electrostatic model. In order to predict the efficiency of the precipitator for each particle size, a differential form of the classical Deutch efficiency equation is used. The precipitator section is divided into fine elements, and the local electric field is used to compute the charging field and the collecting field. The charging field is assumed to be the average field normal to the electrode surface and the collecting field is the field near the collecting electrode at each point consistent with the Deutch model.

It should be recalled that for the purpose of computing the efficiency, the restrictive assumptions of the Deutch model (see, for example, White $(1,2)$ ) are still applicable. These include the assumption that the fluid turbulence is dominant and effective in dispersing the particles to obtain uniform particle concentration in the interelectrode space. Electric field effects are dominant near the collecting electrode. No reentrainment of particulates is allowed, and no particle-particle interactions.

In this section, we describe the basic structure of the model, the boundary conditions, and the techniques of solution which have been employed. The solution in the numerical model employs a finite difference scheme. Since the wavy electrode is not easily represented by a finite difference scheme, a coordinate transformation was employed to map the wavy electrode into a rectangular grid.

Numerical Model

In the present study we have adapted and modified a model for the description of time-averaged fluid motion (.4). The model includes fluid transport equations and electrostatic equations. 
Transport Equations

For time-averaged flow, the continuity and momentum equations can be written as

$$
\begin{gathered}
\frac{\partial \rho U_{i}}{\partial x_{i}}=0 \\
\frac{\partial \rho U_{i} U_{j}}{\partial x_{i}}=-\frac{\partial P}{\partial x_{i}}-\frac{\partial \tau_{i j}}{\partial x_{i}}+\rho * E_{i}
\end{gathered}
$$

where $\rho(x)$ is the time-averaged fluid density at position, $U$ is the averaged velocity, $P$ the pressure, and $\tau_{i j}$ the stress tensor. The term $\rho^{*} E_{i}$ is the pressure gradient caused by the discharge electric field where $\rho^{*}$ is the space charge density, and $E_{i}$ is the electric field intensity. The turbulence kinetic energy equation is written as

$$
\frac{\partial \rho U_{i} k}{\partial x_{i}}=\frac{\partial}{\partial x_{i}} \frac{\mu_{e f f}}{\sigma_{k}} \frac{\partial k}{\partial x_{i}}-\frac{\partial U_{i}}{\partial x_{j}} \tau_{i j}-\rho \varepsilon,
$$

and the turbulence energy dissipation equation as

$$
\frac{\partial \rho U_{i} \varepsilon}{\partial x_{i}}=\frac{\partial}{\partial x_{i}} \frac{\mu_{\text {eff }}}{\sigma_{\varepsilon}} \frac{\partial \varepsilon}{\partial x_{i}}-\frac{\varepsilon}{k}\left(c_{\varepsilon 1} \frac{\partial U_{i}}{\partial x_{j}} \tau_{i j}+c_{\varepsilon 2} \rho \varepsilon\right)
$$

and furthermore

$$
\tau_{i j}=-\mu_{\text {eff }}\left(\frac{\partial U_{i}}{\partial x_{j}}+\frac{\partial U_{i}}{\partial x_{i}}\right)+\frac{2}{3}\left(\rho k+\mu_{e f f} \frac{\partial U_{\ell}}{\partial x_{\ell}}\right) \delta_{i j} .
$$

Equations ( 3 ) and ( 4 ) describe the transport of the turbulent kinetic energy, $k$, and its rate of dissipation, $\varepsilon$. The effective viscosity are given by

$$
\mu_{\text {eff }}=C_{\mu} \rho k^{2} / \varepsilon \text {. }
$$

The parameters $c_{\mu}, C_{\varepsilon l}, C_{\varepsilon 2}, \sigma_{k}$, and $\sigma_{\varepsilon}$ are empirical constants.

\section{Electrostatic Equations}

The two dependent variables describing the electrostatic field are the voltage and the ion space charge. These dependent variables may be described using Maxwell's electrostatic equation and conservation of current.

$$
\begin{array}{ll}
\text { Maxwell's Equation } & \frac{\partial^{2}}{\partial x_{i}^{2}} \phi=-\frac{\rho^{*}}{\varepsilon_{0}} \\
\text { Conservation of Current } & \frac{\partial}{\partial x_{i}}\left(\rho \star b E_{i}\right)=0
\end{array}
$$

where $\phi$ is the electrostatic portential, $\varepsilon_{0}$ is the dielectric constant of the medium, $\rho^{*}$ is the space charge density as before, and $b$ is the mobility assumed to be constant.

These equations model the interaction between the electrostatics and fluld dynamics. However, the model presently excludes the effects of particle dynamics on fluid field using the following assumptions: (1) The response time of any given particle to adjust itself to the surrounding fluid environ- 
ment is sufficiently small to ensure that the particles follow the small-scale fluid motion. (2) In case of low particle loading, the fluid dynamics field exerts little influence on the electric field and, consequently, the correlation between fluid velocity and electric field is neglected.

\section{COMPUTATIONAL MESH GENERATION}

The above set of equations is numerically solved using finite differencing techniques. These computational methods for solving physical problems in complex-shaped regions require the generation of an appropriate grid system in the region. A good grid system ensures the economy and accuracy in the computational procedure. This section will discuss the two independent methods of grid generation: one for the conventional precipitator and the other for the wavy electrode precipitator.

Grid System for Conventional Precipitator

A state-of-the-art conventional precipitator is illustrated in Figure la. An appropriate grid system conforming to this configuration is the one that models the regions of high gradient (in fluid transport and electrostatics) in detail. For this reason, denser grids were allocated to the regions near the wires and the baffles. Additional constraint is imposed on the field intensity near the discharge electrode. Following the discussion in Cobine (6), a characteristic corona radius is used. for the calculations of the field intensity near the wire. The grid system was constructed by taking the first grid node at that characteristic distance away from the wire and gradually increasing the grid size to the center of the precipitator cell. To achieve a better resolution of the velocity gradients near the baffles, a similar method was used in the lateral direction to allocate a denser mesh in those regions. Thus, nonuniform mesh system of 59 x 30 grids was constructed.

\section{Grid System for Wavy Electrode Precipitator}

For the wavy electrode precipitator, a boundary-conforming, orthogonal, curvilinear coordinate system was generated. A recent series of papers by Reid et al. (7) and Mobley and Stewart (8) presented a method with flexibility in generating grid systems more appropriate to physical problems. A second transformation is incorporated to stretch and pack the grid as the problem necessitates.

A sample mesh system of $59 \times 29$ grids for a wavy electrode precipitator was used. The coordinates were packed towards the wire in order to place the first computational grid node at the characteristic wire radius as before. The grids were stretched gradually away from the wire to optimize the number of grid nodes for economical computation.

To utilize the general orthogonal coordinate system, the governing equations were rewritten using the usual rules of coordinate transformation. 
The results of numerical modeling for electrostatics are presented in Figure 2. The applied conditions were a wire voltage of $-70 \mathrm{kV}$ at a room temperature of $15^{\circ} \mathrm{C}$. These conditions were selected for comparisons with laboratory conditions. Obviously, in a full-scale precipitator, the operating temperature will be higher and the maximum allowable operating voltage, as well as the current densities, will be much lower. However, both the conventional and wavy electrodes were considered under the same conditions for our analysis. The test wavy collector was a sine wave with height (from the centerline to the peak of the wave) of $15 \mathrm{~cm}$, wave amplitude (peak-to-valley distance) of $3 \mathrm{~cm}$, and wavelength of $22.5 \mathrm{~cm}$. The solution for the electric potential takes into account the effect of ion space charge, and the boundary conditions along the inlet and outlet are assumed periodic. As can be observed in Figure $2 a$, there is a strong gradient of potential near the baffles in a conventional precipitator, indicating an imminent sparkover under the given test conditions. The wavy electrode shows, on the other hand, more uniform distribution of potential throughout the precipitator cell, as seen in Figure $2 b$. This favorable property of the wavy electrode may allow the applied voltage to be increased without promoting a sparkover as discussed earlier.

Figures $3 a$ and $b$ show the fluid dynamic flow field at corresponding applied conditions. The volume flow rate was set at $0.6 \mathrm{~m}^{3} / \mathrm{s}$ which translated to the inlet velocities of $0.6 \mathrm{~m} / \mathrm{s}$ for the conventional precipitator and $0.5 \mathrm{~m} / \mathrm{s}$ for the wavy electrode precipitator because of varying inlet geometries. As illustrated, the numerical predictions show the dramatic effect of ion motion and ionic wind along the electric field lines.

The influence of the ionic flow is strong enough that just beyond the cell entrance the calculations show a rapid adjustment of the flow to the electric field. The region between the discharge wires experiences a minimum in both the electric field and space charge concentrations; this results in a locally minimum electric pressure gradient $\rho * E_{i}$ which allows the fluid a return path to the wire.

The wavy electrode exhibits a different pattern in separation region near the collection plate. The strong interaction between the ion migration and the fluid motion along the wavy-shaped plate promotes a large recirculation zone behind the inlet section. In the conventional precipitator, the recirculation zone extends to about one-quarter of the cell behind the inlet baffles. On the other hand, nearly two-thirds of the collection plate in the wavy precipitator is protected by the recirculation flows.

A comparison of current flux distribution is given in Figure 4. The applied voltage was at $-70 \mathrm{kV}$ and at room temperature. The solid line corresponds to a numerical prediction of current density of a wavy electrode, and the broken line corresponds to that of a conventional precipitator electrode. The conventional precipitator has a peak current at the plate exactly opposite to the wire, whereas the wavy electrode shows nearly uniform current density over the collection plate. 
This condition is conducive to a higher average migration velocity for the particles being collected. Particularly for fine particles (submicrondiameter particles), the wavy electrode shows favorable trends in improving the particle charging process by two ways: (1) The average charging field over the precipitator cell is higher for the wavy electrode, giving the higher field charging. (2) The average current density in interelectrode space is higher in the wavy electrode, to promote the higher diffusion charging on the fine particles.

The results of these theoretical calculations have been utilized to obtain a qualitative measure of the improvements offered by the wavy design: namely, the increase in particle collection efficiency per unit collector area. Figure 5 shows the efficiency per cell versus particle size using a modified Deutch-Anderson technique. The efficiency predicted in this figure is a relative value and should not be taken as equivalent to the total performance of a given precipitator system. As expected, the submicron particles are most difficult to collect. However, the wavy collector consistently outperforms the conventional collector counterpart. Since the field intensity peaks are not present in the wavy design, sparkover may be delayed and the operating voltage may be increased as schematically illustrated in Figure 5.

\section{CONCLUSIONS}

The advanced precipitator electrode with the wavy geometry shows promising improvements over the conventional precipitator electrode. Based on the present analysis, this improved collector design will offer (1) a higher average electric field and (2) a larger flow-shielding region without sharp flanges. A combination of fluid dynamic advantages and improvements in electrostatic properties may become attractive for precipitator optimization once the improvement provided by each technique is proven. The design may also be incorporated with other design innovations, such as the pulse-charging, rigid-frame electrode and multistaging of precipitators.

\section{ENDNOTES}

1. White, H.J. Industrial Electrostatic Precipitation. Reading, Nassachusetts, Addison-Wesley Publishing Co., Inc., 1963.

2. White, H.J. Electrostatic Precipitation of Fly Ash. Journal of the Air Pollution Control Association, 27(1), January 1977; 27(2), February 1977; 27(3), March 1977; 27(4), April 1977.

3. Robinson, M. Electrostatic Precipitation in Air Pollution Control, Part I., Strauss, W. (ed.). New York, John Wiley \& Sons, Inc., 1971.

4. Bernstein, S. and C.T. Crowe. Interaction Between Electrostatics and Fluid Dynamics in Electrostatic Precipitators. (Presented at the 2nd Symposium on the Transfer and Utilization of Particulate Control Technology, Denver, Colorado, July 23-27, 1979.) 
5. Butler, G. W., K. Ushimaru, and S. Bernstein. An Investigation of Fluid Dynamics, Electrostatics, and Fine Particle Interaction in Electrostatic Precipitators, Flow Research Note No. 187. Kent, Washington, Flow Research Company, December 1980.

6. Cobine, J.D. Gaseous Conductors. New York, McGraw-Hill, 1941.

7. Reid, R.O., A.C. Vastano, R.E. Whitaker, and J.J. Wanstrath. Experiments in Storm Surge Simulation. In: The Sea, Vol. 6, Chap. 5, Goldberg, E.D. (ed.). New York, John Wiley \& Sons, 1977.

8. Mobley, C.D., and R.J. Stewart. On the Numerical Generation of Boundary-Fitted Orthogonal Curvilinear Coordinate Systems. Seattle, Washington, NOAA/ERL Pacific Marine Environmental Laboratory, 1980.

\section{ACKNOWLEDGEMENT}

This effort was supported by the U.S. Department of Energy (DOE) under contract DE-AC03-8EV10506. We wish to acknowledge the encouragement for this effort provided by Dr. Fred Witmer of the DOE.

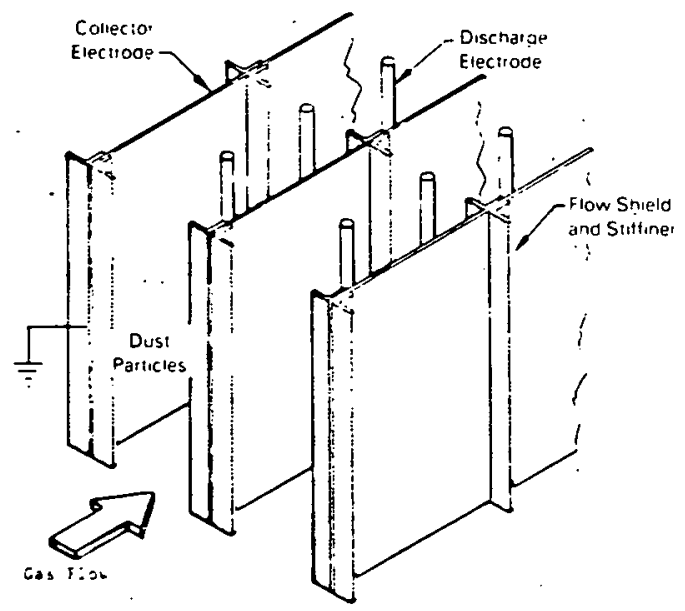

(a) A State-of-the-Art Electrostatic
Precipitator

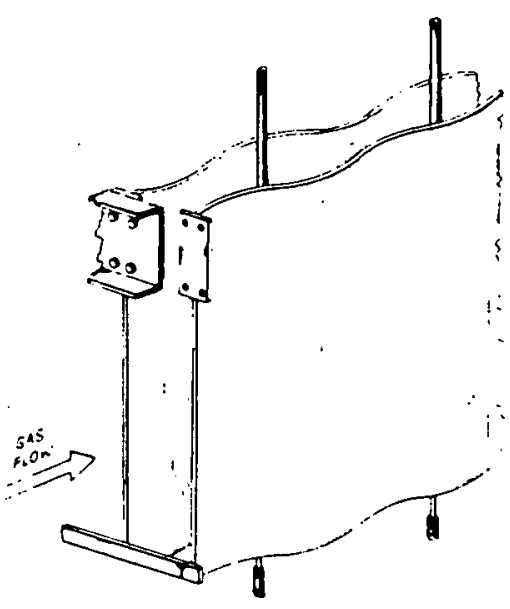

(b) 'The Wavy Electrode Electrostatic Precipitator

Figure 1. Precipitator Electrode Geometry 


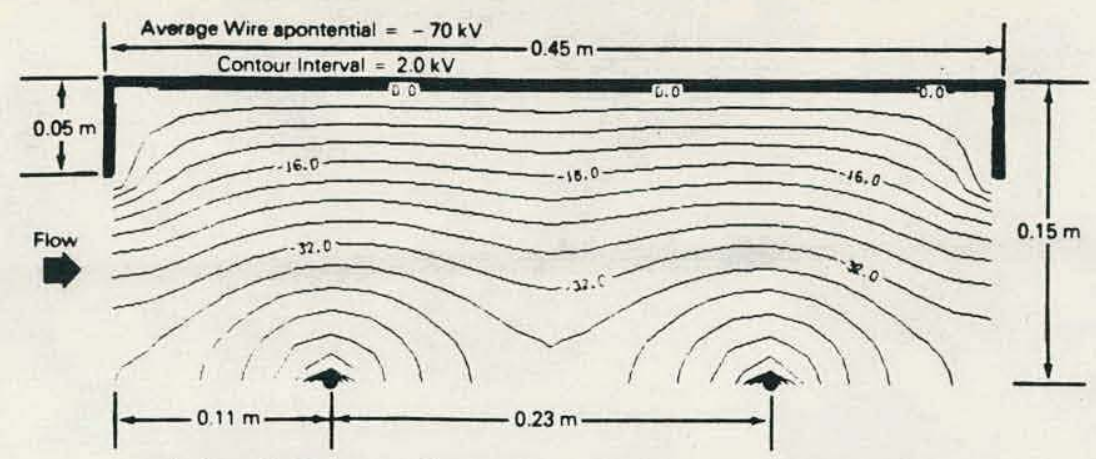

(a) A State-of-the-Art Electostatic Precipitator

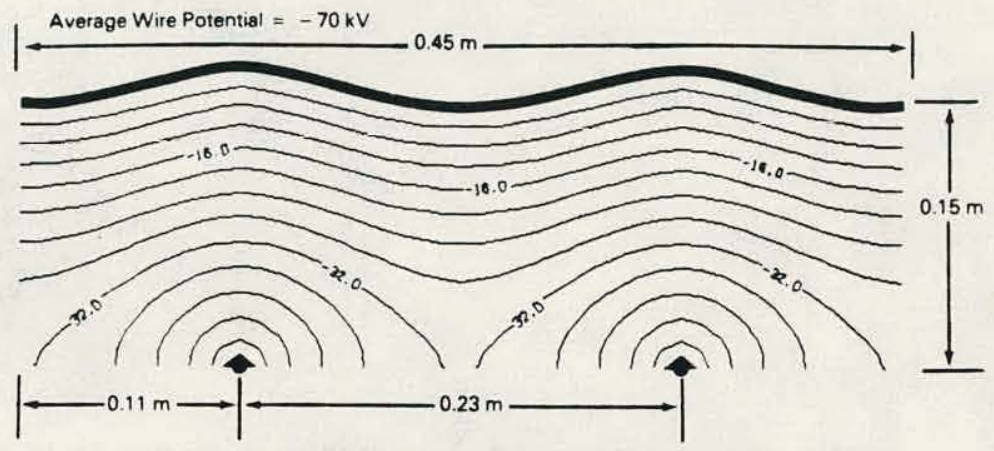

(b) The Wavy Electrode Electrostatic Precipitator

Figure 2. Computed Electrostatic Potential, -70kV

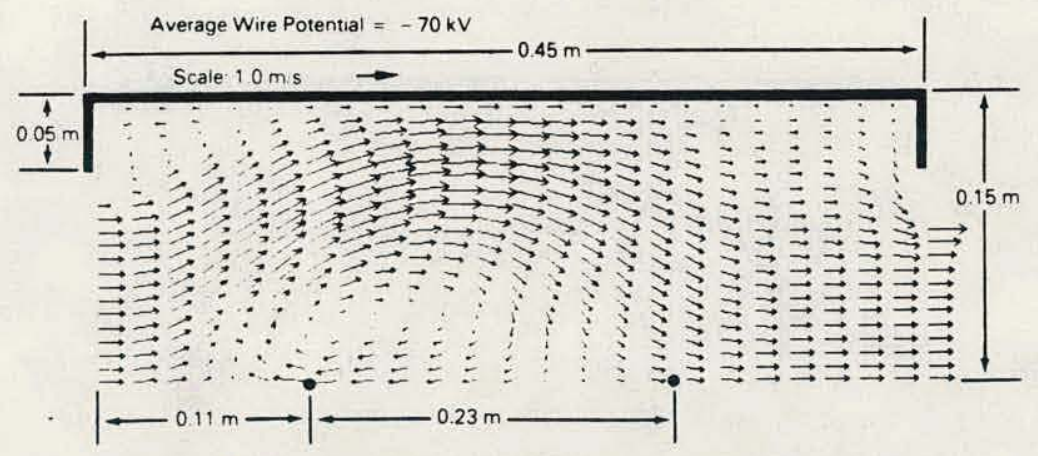

(a) A State-of-the-Art Electrostatic Precipitator

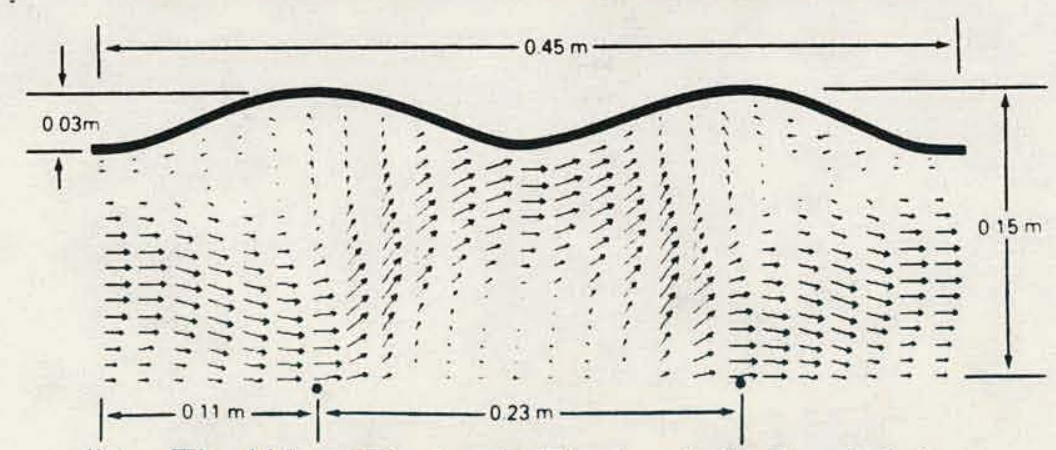

(b) The Wavy Electrode Electrostatic Precipitator

Figure 3. Computed Velocity Field with Average Wire Potential of $-70 \mathrm{kV}$ 


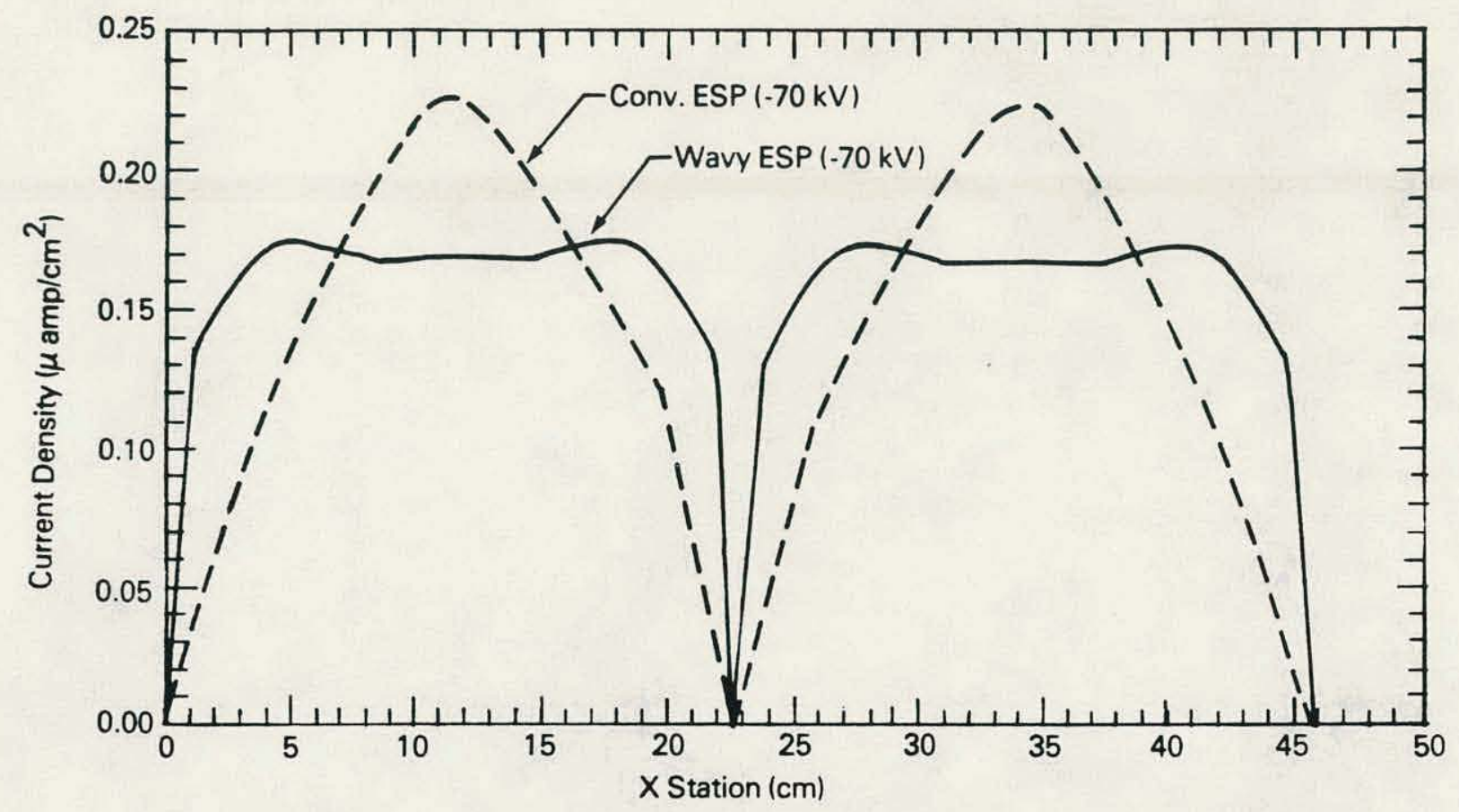

Figure 4. Comparison of Computed Current Density, $-70 \mathrm{kV}$

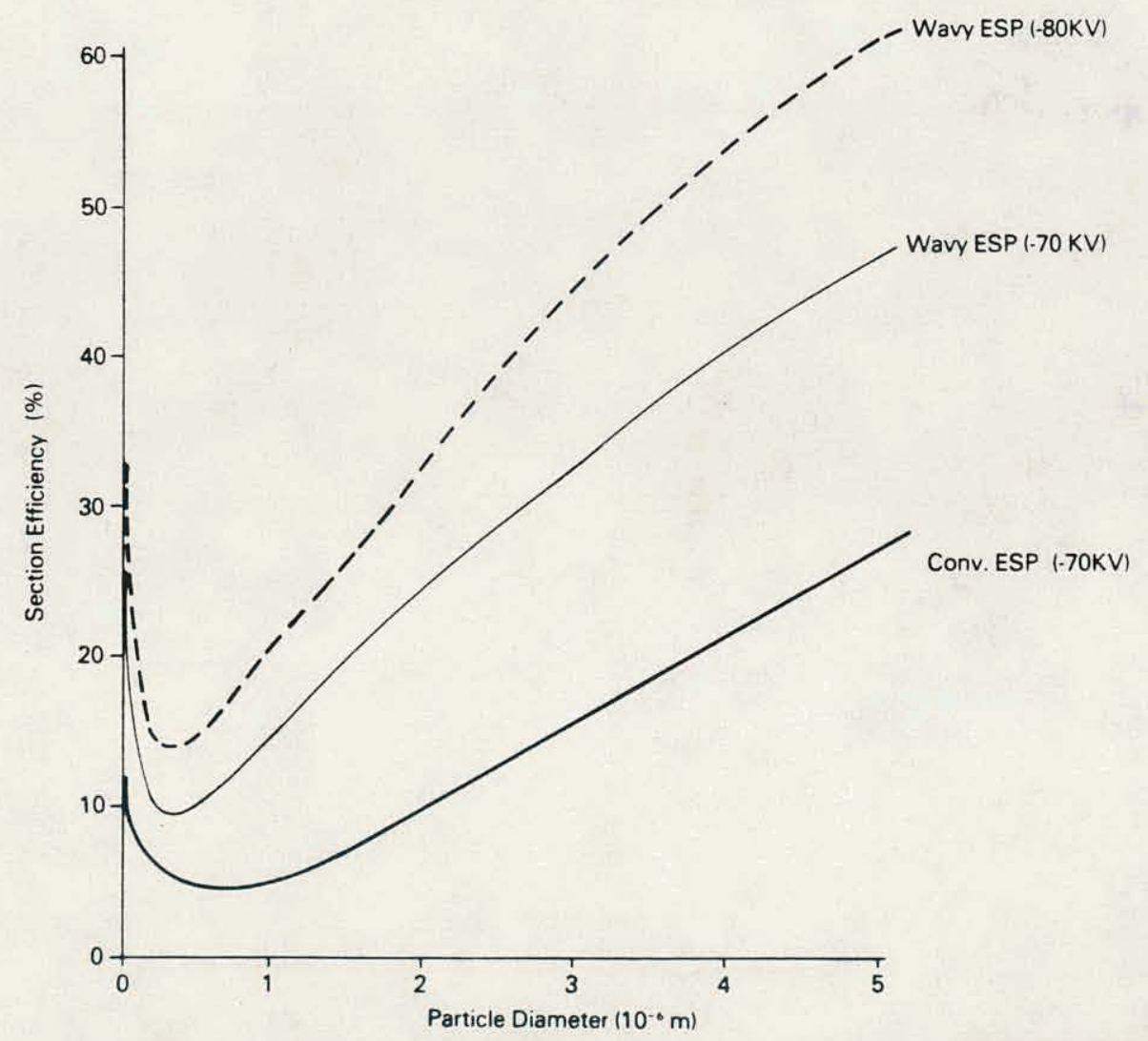

Figure 5. Particle Collection Efficiency as a Function of Particle Size 